\title{
INDIFERENCIA AL TRATAMIENTO DENTAL
}

\section{DENTAL INDIFFERENCE}

\author{
Heredia-Veloz David. ${ }^{1 *}$, Villavicencio-Caparó Ebingen. ${ }^{2}$, Jaramillo-Oyervide Jéssica $^{3}$ \\ ${ }^{1}$ Odontólogo Universidad de Cuenca.Ecuador. \\ 2 Doctor en Ciencias de la Salud, Magíster en Estomatología Especialista en Bioestadística, Coordinador de Investigación de \\ la Carrera de Odontología Universidad Católica de Cuenca. Ecuador. ${ }^{3}$ Magíster en Psicoterapia Integrativa Enfocada en la \\ Personalidad. Docente Universidad Católica de Cuenca. Ecuador. \\ *hdav913@hotmail.com
}

\begin{abstract}
Resumen
Resumen: Revisión bibliográfica que fue realizada con el fin de recolectar información sobre la indiferencia al tratamiento dental, con el propósito de brindar al profesional información relacionada con una de las principales barreras para el acceso a los servicios de salud oral. Las fuentes consultadas para esta revisión fueron artículos científicos de distintas bases digitales de temas relacionados con Odontología, Psicología, Procesos psicoterapéuticos. Se observó que este tipo de conducta es más frecuente en hombres y obreros en edades jóvenes y esta actitud disminuye progresivamente con la edad. Entre los factores de riesgo relacionados con este comportamiento se incluyen el factor socio económico, nivel educacional, adherencia al tratamiento. El método de diagnóstico de esta conducta es la escala de indiferencia dental (DIS) desarrollada por Nuttall en el año 1996. Como tratamiento para la indiferencia dental existen muchas terapias, que no tienen un respaldo estadístico suficiente, pues en Psicología estos procedimientos han sido desarrollados de forma empírica.
\end{abstract}

Palabras clave: Indiferencia dental, Adherencia al tratamiento, Escala de Indiferencia Dental.

\begin{abstract}
Summary:A bibliographic review was done to collect information from dental indifference in order to give information from different articles of the most important obstacles to accesses to oral health service. It is observed that this type of behavior was more frequent in men and workers at young ages and this attitude was progressively decreasing with age; among the risk factors related to this behavior are the socioeconomic factor and emotional states such as fear and anxiety. The method of diagnosis of this behavior is the scale of dental indifference (DIS) developed by Nuttall in 1996. As treatment for dental indifference there are many therapies, which have no statistical support, because in psychology these procedures have been developed from Empirical form.
\end{abstract}

Key words: Dental Indifference, Adherence to treatment, Dental Indifference Scale.

\section{INTRODUCCIÓN}

Entre los principales problemas de salud pública se encuentran las enfermedades bucodentales. Aunque estas afectan una zona específica del cuerpo, sus consecuencias perjudican al organismo en general. ${ }^{1}$ La OMS define como salud bucal "la ausencia de dolor bucal o facial, de cáncer oral o de garganta, de infecciones o úlceras, de enfermedades periodontales, caries, pérdida dentaria, así como de otras enfermedades y alteraciones que limiten la capacidad individual de morder, masticar, reír, hablar o comprometan el bienestar psicosocial". ${ }^{1}$ La salud bucodental ha sido considerada como un derecho básico, aceptado por gran parte de las naciones. ${ }^{1,2}$

Dentro de las patologías prevalentes relacionadas con la cavidad oral destacan la caries dental y enfermedad periodontal. Estudios realizados entre los años 1990 y 2010 reportaron que la caries dental no tratada es la patología más común, entre las 291 enfermedades estudiadas en el marco del Estudio Internacional sobre la carga de las Enfermedades. ${ }^{1,2}$ Esta misma investigación encontró que la enfermedad periodontal, en especial la periodontitis severa, afecta del $5 \%$ al $20 \%$ de la población, ocupando un sexto puesto entre las enfermedades más frecuentes de la boca. Además, este estudio reportó que el décimo puesto de las enfermedades que afectan a la cavidad oral es el cáncer, siendo más frecuente esta patología en el sudeste de Asia debido a los hábitos de la población relacionados con el consumo de alcohol y tabaco. ${ }^{1}$

En Ecuador la patología oral más común es la caries dental, según el estudio Nacional Epidemiológico de Salud Bucal en escolares menores de 15 años realizado en el año 2009 - 2010, encontrándose que el 76,5\% tenían caries dental. En el año 1996 un Estudio Epidemiológico de 
Salud Bucal reflejó que el $84 \%$ de los niños presentaron presencia de placa bacteriana y el $53 \%$ estuvo afectado con gingivitis; se evidenció también que de los niños examinados el $35 \%$ presentaron algún tipo de mal oclusión. Esta situación continúa siendo un problema que requiere pronta solución; sin embargo, gracias a una intervención temprana estas patologías pueden evitarse o tratarse a un costo accesible. Es por ello que el Ministerio de Salud Pública del Ecuador desarrolló un modelo de atención primaria en salud bucodental, el que enfoca las estrategias adecuadas para tratar las patologías en mención. No obstante este modelo no considera los factores emocionales como el miedo y la ansiedad en la consulta odontológica así como tampoco la indiferencia a los tratamientos dentales., ${ }^{2,3}$

Debido a los antecedentes mencionados, las patologías orales han sido un desafío para la salud pública y muchos países han subestimado esta problemática. ${ }^{1}$ La indiferencia al tratamiento dental una de las limitaciones para concluirlo de manera exitosa, lo que ha motivado a realizar una revisión bibliográfica que brinde al profesional de la salud oral conocimientos relacionados a uno de los principales obstáculos para el acceso a los servicios de salud dental. Antes de describir los principales factores de riesgo para la indiferencia dental es muy importante definir conceptos como adherencia terapéutica, la que según Escalante y Granados es una actitud compleja resultado de la combinación de factores conductuales, relacionales y volitivos que dirigen a la participación y comprensión del tratamiento por el paciente y del plan para su cumplimiento, conjuntamente con el profesional de salud para lograr el resultado esperado. A manera de antítesis, se considera la noadherencia al tratamiento como "la falla en el cumplimiento de instrucciones terapéuticas, ya sea de forma voluntaria o inadvertida, la negativa en seguir los esquemas de tratamiento indicados" siendo una decisión personal multifactorial. ${ }^{4}$

La indiferencia al tratamiento dental es una "actitud definida como una subestimación de los dientes o falta de interés en la salud oral" ${ }^{2}$ manifestada principalmente por poco cuidado dental, falta de cumplimento en recomendaciones del profesional, el escaso compromiso en la asistencia a las citas odontológicas debido a diversos juicios errados como restarle importancia a estos procedimientos o escoger el tratamiento dental no adecuado o el más rápido según el criterio del paciente. ${ }^{5,6}$

Distintas investigaciones han utilizado como instrumento de medida de esta conducta la Escala de Indiferencia Dental desarrollada por Nuttall (TABLA 1), la misma que se caracteriza por ser un instrumento rápido, sencillo y preciso para la actitud que evalúa. ${ }^{7}$

\subsection{FACTORES DE RIESGO DE LA INDIFERENCIA DENTAL}

La literatura manifiesta que algunos pacientes realizan un análisis de riesgo-beneficio de la necesidad o las ventajas de seguir o no un tratamiento. Con frecuencia la decisión del paciente se basa en la evaluación de la importancia considerada en cuatro factores:

1) La prioridad de cumplir con el tratamiento.

2) La percepción de gravedad de la enfermedad.

3) La credibilidad de la eficacia del tratamiento.

4) Aceptación personal de los cambios recomendados.

Diversas investigaciones han encontrado varias causas relacionadas con la indiferencia dental. ${ }^{8,9}$ Un estudio realizado en Reino Unido en 1978 reveló que los factores de riesgo más frecuentes en la población dentada para no asistir a la cita odontológica era el miedo y la ansiedad; por otro lado en una investigación realizada en Escocia se corroboró que la apatía al tratamiento dental era una de las razones más frecuentes, al igual que el miedo, para evitar acudir a la consulta dental, aunque esta razón variaba según cada género. ${ }^{6,10}$

Entre otros factores de riesgo para la indiferencia dental, se menciona el nivel socioeconómico; pues este limita al paciente para asistir a consultas periódicamente, o realizar tratamientos adecuados según cada patología. ${ }^{5}$ Una investigación realizada en Suecia, ${ }^{6}$ demostró que el nivel socioeconómico bajo estaba relacionado directamente con la indiferencia dental en especial dentro de la atención odontopediátrica. En Brasil $^{8}$ se observó que la actitud de indiferencia a la atención dental también se relacionaba con las limitaciones económicas, siendo el grupo más afectado los adolescentes.

El nivel de educación y preparación de los padres es otro factor influyente en esta actitud de indiferencia, especialmente en niños y adolescentes, según manifestó una investigación realizada en Suecia, donde se observó que el número de citas dentales canceladas y tratamientos abandonados fueron más frecuentes en padres desempleados y con bajo nivel de preparación que en hijos de padres preparados y con un trabajo estable. ${ }^{5}$

Es muy importante mencionar que existen factores de riesgo asociados con el paciente y con otras patologías en general, que son limitantes para adherencia terapéutica, ocasionando de esta forma indiferencia a los tratamientos dentales; entre estas complicaciones sistémicas se mencionan deterioro sensorial, cognoscitivo y estados alterados de ánimo, pues muchos pacientes se acostumbran a la sintomatología de la patología y prefieren tolerar dicho padecimiento antes que cumplir el tratamiento. ${ }^{4}$

\subsection{FRECUENCIA DE LA INDIFERENCIA DENTAL.}

Diversos estudios han demostrado que la indiferencia dental es más frecuente en hombres jóvenes y obreros, ya que este grupo se caracteriza por el abandono y escasa asistencia a las consultas dentales o asisten cuando requieren algún 


\begin{tabular}{|c|c|c|}
\hline PREGUNTA & RESPUESTA & PUNTUACION \\
\hline 1. & $\begin{array}{l}\text { Usualmente uso (marque cualquiera que aplique) } \\
\text { a. Un cepillo dental para limpiar mis dientes. } \\
\text { b. Seda o un cepillo especial para limpiar entre mis dientes. } \\
\text { c. Tabletas para comprobar que mis dientes están limpios. }\end{array}$ & $\begin{array}{l}\text { Valor: } 1 \text { si ninguno o si ni el literal b ni } \\
\text { literal c están marcados }\end{array}$ \\
\hline 2. & $\begin{array}{l}\text { En la actualidad: } \\
\text { a. Creo que hay algo mal con mis dientes, pero no es lo } \\
\text { b. Creo que hay algo mal con mis dientes. Y tengo la } \\
\text { intención de ver a un dentista pronto. } \\
\text { c. Voy a hacerme un chequeo el próximo año } \\
\text { d. No creo que necesite ningún tratamiento por lo que no }\end{array}$ & $\begin{array}{l}\text { Valor: } 1 \text { si el literal a o d están } \\
\text { marcados }\end{array}$ \\
\hline 3. & $\begin{array}{l}\text { Si perdi una restauración en un diente, pero no me dolió. } \\
\text { a. De inmediato iré a un dentista. } \\
\text { b. Esperaría si siento dolor o algún síntoma antes de ir a } \\
\text { c. No dentista. } \\
\text { razón }\end{array}$ & $\begin{array}{l}\text { Valor : } 1 \text { si el literal b o c están } \\
\text { marcados }\end{array}$ \\
\hline 4 & $\begin{array}{l}\text { Por lo general, hago una cita para visitar a un dentista } \\
\text { a. Cuando mi dentista me lo recuerda. } \\
\text { b. Al final de mi última cita. } \\
\text { c. Cuando pienso que es tiempo de ir a otro chequeo. } \\
\text { d. Unicamente cunado pienso que esta algo malo en mis } \\
\text { dientes. }\end{array}$ & Valor: 1 si el literal d está marcado \\
\hline 5. & $\begin{array}{l}\text { Si mis encías sangran, pero estas no duelen } \\
\text { a. No es un problema no vería a un dentista por este } \\
\text { broblema. } \\
\text { b. Iría inmediatamente a una cita con el dentista. } \\
\text { c. Esperaría a ver si se presenta dolor o algún síntoma } \\
\text { para ir al dentista }\end{array}$ & $\begin{array}{l}\text { Valor: } 1 \text { si el literal a o c están } \\
\text { marcados }\end{array}$ \\
\hline 6. & $\begin{array}{l}\text { Acerca de todas sus citas dentales en los últimos } 5 \text { años } \\
\text { marque la más cercana. } \\
\text { a. No he tenido una cita dental en los últimos } 5 \text { años. } \\
\text { b. Durante los últimos } 5 \text { años he olvidado de asistir a mis } \\
\text { c. Durante los últimos } 5 \text { años solo he perdido una sola } \\
\text { c. Durante los últimos } 5 \text { años nuca he perdido una cita } \\
\text { e. Dental. } \\
\text { e. Durante los últimos } 5 \text { años he cancelado una cita } \\
\text { dental debido a que el problema desapareció }\end{array}$ & $\begin{array}{l}\text { Valor: } 1 \text { si el los literales a, b o e están } \\
\text { marcados }\end{array}$ \\
\hline 8. & $\begin{array}{l}\text { Si tuviera un diente muy doloroso: } \\
\text { a. Preferiría que me lo saquen. } \\
\text { b. Preferiría dejarlo sin tratamiento } \\
\text { c. Preferiría realizar una restauración. } \\
\text { Yo diría que mi razón principal para no ir a un dentista para } \\
\text { un chequeo sería. }\end{array}$ & $\begin{array}{l}\text { Valor: } 1 \text { si los literales a o b están } \\
\text { marcados. } \\
\text { Valor: } 1 \text { si los literales b, d ó g están } \\
\text { marcados }\end{array}$ \\
\hline & $\begin{array}{l}\text { a. Porque pienso que el tratamiento es doloroso. } \\
\text { b. Porque toma demasiado tiempo ir al dentista. } \\
\text { c. Porque siento preocupación o ansiedad cuando } \\
\text { voy al dentista. } \\
\text { d. Porque mi dentista me hace sentir culpable } \\
\text { e. Porque cuestado de mis dientes } \\
\text { f. Porque no tengo tiempo de ir al dentista. } \\
\text { g. No pospongo las citas. Yo asisto al dentista } \\
\text { regularmente }\end{array}$ & $\begin{array}{l}\text { Si el literal c está marcado no es un } \\
\text { puntaje de indiferencia dental. }\end{array}$ \\
\hline
\end{tabular}

Tabla 1. ESCALA DE INDIFERENCIA DENTAL (DIS) Traducido de: Nuttall NM; Initial development of a. scale to measure dental indifference. Community Dent Oral Epidemiology 1996; 24; 112-6.

tipo de intervención emergente; sumada a esta situación se encuentra un alto nivel de dientes perdidos en este grupo, por lo cual encajarían perfectamente en la categoría de pacientes con indiferencia al tratamiento dental. ${ }^{6,11}$ Sumadas a estas características, este grupo de pacientes presentaron un bajo cumplimiento a las recomendaciones de salud y poca motivación; además se cree que este nivel de indiferencia al tratamiento dental estaría relacionado con las condiciones sociales y económicas ya que para esta clase de personas existen otras necesidades más importantes que sus piezas dentales. ${ }^{12}$

Un estudio realizado en Noruega reportó que pacientes varones de 18 años de edad cancelaban sus citas con el dentista, demostrando así una indiferencia al tratamiento. La prevalencia de este tipo de comportamiento incrementó en hombres de edades entre 12 a 18 años por lo cual esta actitud se reflejó en pérdida de piezas dentales a temprana edad, siendo este problema más frecuente en varones que en mujeres (TABLA 2). ${ }^{8,13}$

Varias investigaciones realizadas en Reino Unido han 


\begin{tabular}{|c|c|c|c|c|c|}
\hline Referencia & País & Instrumento & Sexo & Edad & $\begin{array}{l}\% \text { de la } \\
\text { indiferencia } \\
\text { dental }\end{array}$ \\
\hline Nutall 1996 & Escocia & DIS & Masculino & $\begin{array}{l}\text { Mayores } 30 \\
\text { años. }\end{array}$ & $66.3 \%$ \\
\hline Nutall 1996 & Escocia & DIS & Masculino & $\begin{array}{l}\text { Hombres } \\
\text { jóvenes de } 21 \text { - } \\
29 \text { años }\end{array}$ & $25 \%$ \\
\hline Nutall 1996 & Escocia & DIS & $\begin{array}{l}\text { Masculino } \\
\text { (obreros) }\end{array}$ & Obreros & $55.3 \%$ \\
\hline $\begin{array}{l}\text { Skaret } 2010 \\
\text { Marshaman } \\
2014\end{array}$ & $\begin{array}{l}\text { Noruega } \\
\text { Inglaterra }\end{array}$ & $\begin{array}{l}\text { DIS } \\
\text { DIS }\end{array}$ & $\begin{array}{l}\text { Masculino } \\
\text { Masculino }\end{array}$ & $\begin{array}{l}18 \text { años. } \\
\text { Hombres } \\
\text { jóvenes de 16- } \\
24 \text { años }\end{array}$ & $\begin{array}{l}83.2 \% \\
73.5 \%\end{array}$ \\
\hline
\end{tabular}

Tabla 2. Frecuencia de la Indiferencia Dental.

encontrado que el grupo etario con mayor frecuencia en mostrar indiferencia al tratamiento dental son adultos jóvenes, especialmente varones, cuyas edades oscilan entre 16 a 24 años.Aunque la indiferencia dental va disminuyendo progresivamente con la edad, esta investigación realizada en personas adultas demostró que estos pacientes aún contaban con piezas dentales y que la pérdida de dientes ocurrió en edades tempranas. ${ }^{6,13}$

El abandono a las citas dentales ha sido identificado también como una actitud que refleja la indiferencia al tratamiento dental. En países nórdicos a pesar que niños y adolescentes tienen gratuidad a servicios dentales independientemente del estado socio económico o la cobertura del seguro, se ha comprobado un gran abandono de tratamientos y citas dentales especialmente en adolescentes de sexo masculino, situación que se observó tanto en el sector público como privado. ${ }^{10}$

En Finlandia, Estados Unidos, Uganda y Canadá la visita dental anual fue menor en los adolescentes. Un estudio realizado en Estados Unidos mencionó que una de las causas de abandono dental fue que el idioma utilizado en las citas odontológicas fue el inglés, y ciertos pacientes que pertenecían a grupos de migrantes lo entendían poco, lo que causaba una relación profesional - paciente deficiente. ${ }^{10}$

\subsection{MEDICIÓN DE LA INDIFERENCIA DENTAL (ESCA- LA DIS)}

En 1996 Nuttall en Escocia desarrolló un cuestionario, cuyo fin fue identificar y medir las actitudes de indiferencia al tratamiento dental.Este cuestionario excluyó pacientes que no asistían a la consulta odontológica por motivos relacionados al miedo y la ansiedad.$^{5-8,13}$ Este cuestionario se caracteriza por ser rápido y corto debido a la actitud que evalúa; esta prueba también es empleada para identificar grupos que requieren promoción en salud oral. El reto más exigente que presenta la escala (DIS) es determinar si es un medio fiable para identificar comportamientos que se asocian con una actitud de indiferencia hacia el tratamiento odontológico como la falta de cumplimiento con las instrucciones de atención oral y la inasistencia a citas dentales. El creador de esta escala propuso utilizar este instrumento conjuntamente con la escala de ansiedad dental, para que ambas escalas permitan determinar el comportamiento y actitud de los pacientes hacia los tratamientos dentales. ${ }^{6}$

Según esta escala, se clasificó a la indiferencia dental en tres tipos: baja (puntuación de 0 a 1), media (puntuación de 2 a 4) y alta (puntuación de 5 a 8). Cabe recalcar que las puntuaciones fueron sumadas según la escala de Nuttall, lo que brindó niveles de seguridad y fiabilidad. ${ }^{6,13}$

En Noruega realizaron una adaptación de este cuestionario a pacientes de 16 a 18 años, pero esta escala no brindó niveles de fiabilidad adecuados. Esto se debió, principalmente, a la existencia de diferentes hábitos orales y distintas prestaciones de servicios de salud oral de Escocia que es el país de origen de la escala, en comparación con Noruega. ${ }^{8}$

\section{TRATAMIENTO DE LA INDIFERENCIA.}

Al considerarse la indiferencia como respuesta de la no adherencia al tratamiento, resulta interesante plantear el tratamiento o la superación de esta barrera conductual, analizando las razones por la que se presenta:

- Razones asociadas al paciente: (Edad, entorno cultural y social, nivel de educación, personalidad,) pueden condicionar el tratamiento; además la desconfianza de que las indicaciones, dadas por el profesional de salud, no sean las adecuadas, que el medicamento no sea efectivo, o que las explicaciones sobre la enfermedad y el tratamiento hayan sido insuficientes o difíciles de entender. Otras veces se puede asociar como causa los olvidos, limitaciones debido a problemas afectivos y cognitivos. ${ }^{14}$ 


\begin{tabular}{|c|c|c|c|c|}
\hline Paciente & $\begin{array}{l}\text { Estrategia de } \\
\text { resolución }\end{array}$ & Profesional de la salud. & Problema & Autores \\
\hline $\begin{array}{l}\text { Mira el tratamiento como } \\
\text { algo complejo }\end{array}$ & Técnicas. & $\begin{array}{l}\text { Simplificar el tratamiento, procedimientos sencillos, } \\
\text { explicación acorde al nivel del paciente. }\end{array}$ & $\begin{array}{l}\text { Indiferencia. } \\
\text { No adherencia } \\
\text { al tratamiento. }\end{array}$ & $\begin{array}{l}\text { Dilla y colbs. } \\
2009 / \text { Ortego } \\
\text { y colbs } 2011 .\end{array}$ \\
\hline $\begin{array}{l}\text { Asume el tratamiento como } \\
\text { observador externo. }\end{array}$ & Conductuales. & $\begin{array}{l}\text { Otorga al paciente responsabilidad sobre el } \\
\text { tratamiento. Reforzamiento positivo por cada etapa o } \\
\text { cumplimiento realizado. }\end{array}$ & $\begin{array}{l}\text { Indiferencia. } \\
\text { No adherencia } \\
\text { al tratamiento. }\end{array}$ & $\begin{array}{l}\text { Dilla y colbs. } \\
2009 / \text { Ortego } \\
\text { y colbs } 2011 .\end{array}$ \\
\hline $\begin{array}{l}\text { No conoce el problema de } \\
\text { salud que padece y las } \\
\text { complicaciones que implica. }\end{array}$ & Educativas. & $\begin{array}{l}\text { Aproximaciones didácticas, lograr que el paciente } \\
\text { asuma una actitud positiva mediante el conocimiento } \\
\text { amplio de su padecimiento y de la importancia que } \\
\text { implica el cumplimiento para su recuperación. }\end{array}$ & $\begin{array}{l}\text { Indiferencia. } \\
\text { No adherencia } \\
\text { al tratamiento. }\end{array}$ & $\begin{array}{l}\text { Dilla y colbs. } \\
2009 / \text { Ortego } \\
\text { y colbs } 2011 .\end{array}$ \\
\hline $\begin{array}{l}\text { No tiene la capacidad de } \\
\text { pedir ayuda. }\end{array}$ & $\begin{array}{l}\text { Apoyo familiar y } \\
\text { social. }\end{array}$ & $\begin{array}{l}\text { Intervención con familiares para que conozcan sobre la } \\
\text { enfermedad y el tratamiento, }\end{array}$ & $\begin{array}{l}\text { Indiferencia. } \\
\text { No adherencia } \\
\text { al tratamiento. }\end{array}$ & $\begin{array}{l}\text { Dilla y colbs. } \\
2009 / \text { Ortego } \\
\text { y colbs } 2011 .\end{array}$ \\
\hline $\begin{array}{l}\text { Temeros y limitado en la } \\
\text { expresión de sus emociones } \\
\text { por sentirse y estar enfermo. }\end{array}$ & $\begin{array}{l}\text { Profesional de la } \\
\text { salud. }\end{array}$ & $\begin{array}{l}\text { Profesional con destrezas en comunicación efectiva, } \\
\text { manejo conductual adecuado con pacientes que } \\
\text { muestren indiferencia al tratamiento. }\end{array}$ & $\begin{array}{l}\text { Indiferencia. } \\
\text { No adherencia } \\
\text { al tratamiento. }\end{array}$ & $\begin{array}{l}\text { Dilla y colbs. } \\
2009 \text { / Ortego } \\
\text { y colbs } 2011 .\end{array}$ \\
\hline Sentirse fracasado. & $\begin{array}{l}\text { Prevención de } \\
\text { recaídas. }\end{array}$ & $\begin{array}{l}\text { Considerar razones de recaídas y asumirlas como } \\
\text { parte del proceso de tratamiento, no como fracaso. }\end{array}$ & $\begin{array}{l}\text { Indiferencia. } \\
\text { No adherencia } \\
\text { al tratamiento. }\end{array}$ & $\begin{array}{l}\text { Dilla y colbs. } \\
2009 / \text { Ortego } \\
\text { y colbs } 2011 .\end{array}$ \\
\hline
\end{tabular}

Tabla 3. Estrategias para disminuir indiferencia y lograr adherencia al tratamiento.

- Razones asociadas al tratamiento: cuando se usan medicamentos, se consideran los efectos secundarios adversos que producen, el tiempo en el que se ven los resultados beneficios. Los pacientes esperan resultados a corto plazo, la complejidad del régimen terapéutico dada por las prescripciones o las etapas del tratamiento, los costos en Odontología que pueden ser altos, si el problema que se presenta es agudo o crónico elevando el tiempo y el precio del tratamiento. ${ }^{14}$

- Razones asociadas al profesional de la salud: se considera el tiempo que se invierte en cada paciente para el tratamiento, dificultades del profesional para comunicarse con el paciente, la personalidad del profesional, el conocimiento sobre el problema de salud oral que se presenta y la efectividad con que pueda manejar las reacciones y cuestionamientos del paciente y del tratamiento. ${ }^{14}$

Orego 2011 en su estudio de adherencia al tratamiento coincide que para analizar esta temática hay que considerar: Variables sociodemográficas (edad, sexo, nivel sociocultural, ocupación, y número de hijos o adultos a cargo, etc,) creencias del paciente, refiere que cada paciente tiene sus representaciones de lo que significa las amenazas para la salud y planifican según estas representaciones y temores que le acompañan, el tipo de problema de salud, el tratamiento que se plantea, la relación entre paciente y profesional de la salud, el apoyo familiar y social que el paciente tenga, entre las más relevantes. ${ }^{15}$

En sintésis tenemos como principal estrategia entrenar a los pacientes en el tratamiento y con ellos a los familiares o allegados que sean de importancia y que apoyen el cumplimiento de manera efectiva considerando también: técnicas enfocadas en simplificar el tratamiento, recomendación de procedimientos sencillos, explicados acorde al nivel de las posibilidades de entendimiento del paciente:

Conductuales: en las que se busca estrategias de cumplimiento otorgando al paciente responsabilidad sobre el tratamiento. Por ejemplo: cepillo de dientes en el trabajo, recordatorios para que no olvide su salud oral por la noche, calendarios amplios con registros de las fechas establecidas para el tratamiento, recompensas o reforzamiento positivo por cada etapa o cumplimiento realizado.

Educativas: aproximaciones didácticas enfocadas en lograr que el paciente asuma una actitud positiva frente al tratamiento mediante el conocimiento amplio de su padecimiento y de sus posibles complicaciones, de la importancia que implica el cumplimiento para su recuperación, asegurarse que el paciente haya entendido a profundidad su situación de salud, con esto garantizamos que la adherencia al tratamiento sea una realidad.

Apoyo familiar y social: intervención con familiares 


\begin{tabular}{lll}
\hline Referencia & Tratamiento & Efectividad \\
\hline Little (1997) & $\begin{array}{l}\text { No pudo ser } \\
\text { Evaluada por } \\
\text { Abandono de } \\
\text { stewart (1991) }\end{array}$ & $\begin{array}{l}\text { Teoría del aprendizaje social } \\
\text { Teoría de aprendizaje social }\end{array}$ \\
& $\begin{array}{l}\text { (Evaluación } \\
\text { pre-intervención a los 6 meses) } \\
\text { Teoría de aprendizaje ( Evaluado } \\
\text { al inicio, a los 30 días y 60 días } \\
\text { después de la línea de base) }\end{array}$ & $\begin{array}{l}\text { Ningún resultado } \\
\text { estadísticamente } \\
\text { significativo }\end{array}$ \\
\hline
\end{tabular}

Tabla 4. Efectividad de tratamiento en indiferencia dental.

para que conozcan sobre la enfermedad y el tratamiento, lo cual genera que se conviertan en apoyos efectivos para cuando el paciente de indicios de abandono del tratamiento.

Profesionales de la salud: instruir para que el profesional adquiera destrezas en comunicación efectiva, habilidades de manejo conductual ante pacientes que muestren actitudes o conductas de indiferencia al tratamiento, el odontólogo debe tener la suficiente habilidad para crear un ambiente de confianza en el que el paciente pueda expresar sus temores, desconfianzas y preocupaciones acerca de su tratamiento, considerar además sus rasgos de personalidad para que el acercamiento sea efectivo.

Prevención de recaídas: Que el paciente sepa que esta posibilidad existe implica también que esté prevenido y las evite. Considera las razones por la que se dio la recaída y trabajar en ellas como parte del proceso de tratamiento, mas no como un fracaso. ${ }^{14,15}$

Existen distintas estrategias empleadas dentro de la Psicología, cuyo fin tiene disminuir la indiferencia y lograr una adherencia al tratamiento. Lamentablemente no existen datos estadísticos suficientes que permiten hacer inferencia sobre el éxito de estas terapias.

\section{ESTRATEGIAS PARA DISMINUIR INDIFEREN- CIA Y LOGRAR ADHERENCIA AL TRATAMIEN- TO DENTAL.}

En la revisión sistemática realizada por Renz en el año 2007, se mencionó que desde los años 70 ha existido un gran interés por los psicólogos en los factores que modifican el comportamiento humano. Entre estos destacaron los modelos de cognición social los cuales han sido ampliamente utilizados en la modificación de conductas, incluida la higiene dental. En la década de los 90 los conocimientos aportados por estos modelos cognitivos fueron utilizados para realizar intervenciones capaces de modificar el comportamiento humano. ${ }^{16}$

Little en el año 1997 adoptó la Teoría del Aprendizaje Social en un grupo de 107 pacientes de 50 a 70 años con enfermedad periodontal moderada. La muestra de este grupo estuvo conformada por 53 personas que recibieron terapia periodontal y de mantenimiento; se les impartió conocimientos sobre aspectos básicos de la enfermedad, recibiendo 5 sesiones informativas de 90 minutos de duración cada una. Se realizó el control luego de 4 meses y cinco de los participantes abandonaron el tratamiento, por esta razón la validez de la teoría no pudo ser evaluada. ${ }^{16}$

Stewart en el año de 1991 investigó en un grupo conformado por 100 hombres de edades comprendidas entre 21 a 65 años de los cuales tenían enfermedad periodontal. El grupo se dividió en 4 subgrupos con el mismo número de pacientes a quienes se le impartieron charlas informativas en cepillado dental y uso de hilo dental de una duración de 20 minutos. La intervención cognitiva del comportamiento fue realizada por un psicólogo que utilizaba refuerzo verbal simple para superar las barreras de comportamiento. (ver tabla 4)

\section{CONCLUSIONES}

Luego de la revisión bibliográfica se concluye:

- Entre los principales factores de riesgo de este comportamiento se relacionan los socioeconómicos, culturales y el nivel de preparación de cada paciente, así como la capacidad de afrontar conflictos.

- El grupo mayormente afectado por esta conducta está representado por hombres en edad joven. 
- La indiferencia dental es una conducta que se presenta en algunos grupos de pacientes, la misma que ha sido considerada como una de las principales barreras para el acceso y desarrollo de la terapia dental. Aunque determinarla es difícil y varía según cada región y género se ha utilizado la DIS, un método empleado por distintas investigaciones que ha sido calificado como un procedimiento útil y rápido para visibilizar este tipo de comportamiento.

- La indiferencia, no es un producto del poco interés del paciente hacia el tratamiento odontológico, o de una conducta de indiferencia elemental. Por el contrario, es resultado de múltiples factores como: falta de personal de salud que brinde al paciente desde el inicio del proceso la sensación de sentirse acogido y entendido en su problemática, la actitud empática generada del profesional de salud y los mecanismos de afrontamiento que el paciente tiene para conducirse en situaciones que le causan tensión emocional. Por lo que hay que considerar que si realizamos nuestro trabajo tomando en cuenta los postulados indicados, la indiferencia puede convertirse en una reacción leve y pasajera. Por lo tanto se recomienda a los profesionales de la salud ir más allá d la descalificación instantánea frente a la indiferencia del paciente, considerándola a esta como una reacción de varios elementos que se deben entender y superar.

Conflicto de intereses y financiamiento Los autores declaran no tener conflicto de intereses, haber cumplido con los requisitos de autoría y haber autofinanciado este artículo.

\section{Referencias}

1 Federación Dental Internacional .El Desafío de las Enfermedades Bucodentales - Una llamada a la acción global. Atlas de Salud Bucodental. $2^{\text {a }}$ ed. Ginebra: Federación Dental Internacional (FDI); 2015.

2 Ministerio de Salud Pública. Protocolos odontológicos. 1.a ed. Quito: Programa Nacional de Genética y Dirección Nacional de Normatización, 2013. Disponible en: http://salud.gob.ec

3 Ministerio de Salud Pública del Ecuador. Plan Nacional de Salud Bucal. Proceso de Normatización del SNS Área de Salud Bucal. 2a ed. Quito: Junio 2009

4 Silva G E, Galeano E, Correa J O, Adherencia al tratamiento. Implicaciones de la no-adherencia. Acta Médica Colombiana 2005 30268-273. Disponible en: .

5 Marshman Z, Baker SR, Robinson PG. Does dental indifference influence the oral health-related quality of life of prisoners? Community Dent Oral Epidemiol 2014; 42: 470-480.

6 Nuttall NM; Initial development of a .scale to measure dental indifference. Community Dent Oral Epidemiol 1996; $24 ; 112-6$.
7 Schuirs AHB. HoociTRATtN J. Appraisal of dental anxiefy and fear questionnaires: a review. Communitv Dem Orat Epidemiol 1993; 21: 329-39.

8 Skaret E, Raadal M, Kvale G, Berg E: Reliability and validity of the Dental Indifference Scale in a population of 18 -year-olds in Norway. Community Dent Oral Epidemiol 2000; 28: 330-5.

9 Iter Horst G, de Wit CA. Review of behavioral research in dentistry 1987-1992: dental anxiety, dentist-patient relationship, compliance and attendance. Int Dent J 1993; 43:265-278.

0 Craven R. Blinkhorn AS, St Nou L. A campaign encouraging dental attendance among adolescents' in Scotland: the barriers for behavior change. Coniniunitv Dent Health 1994; 131-134.

11 Corah NL. Development of a dental anxiety scale. J Dent Res 1969; 48: 596.

12 Camner LG. SandilL R, Sarged G. The role of palient involvement in oral hygiene compliance. Br J Clin Psvcliol 1994; 33:379 90

13 López Garvi Antonio. Estudio de la satisfacción del paciente en odontología mediante cuestionarios de salud: adaptación al español del cuestionario "dental satisfaction questionnaire”. Tesis doctoral .valencia España 2012: 1-200.

14 Dilla, T. Valladares, A. Lizán, L. y Sacristán, J. Adherencia y persistencia terapéutica: causas, consecuencias y estrategias de mejora. Elsevier. 2009. .

15 Ortego, M. López, S. Álvarez, M. Adherencia al tratamiento. Universidad de Cantabria. Ciencias de la Salud. 2011.

16 Renz A, Ide M, Newton T, Robinson P, Smith D. Psychological interventions to improve adherence to oral hygiene instructions in adults with periodontal diseases. Cochrane Database of Systematic Reviews 2007.

Recibido: 16 de Julio de 2017. Aceptado: 30 de Octubre de 2017. 
